\title{
Mecânica Clássica: Posição e Distância
}

\author{
Mario Cezar Bertin ${ }^{1}$ \\ ${ }^{1}$ Instituto de Física, Universidade Federal da Bahia
}

24 de setembro de 2020

Rich media available at https://youtu.be/9Ip7-00a8UM

\section{Resumo dos conceitos primitivos}

Vamos relembrar os conceitos primitivos que apresentamos em aulas passadas, para preparar o terreno do que vem a seguir.

Primeiro, introduzimos o conceito de partícula clássica, que consiste em um objeto sem dimensões, que não pode ser criado ou destruído, e que possui um conjunto de observáveis associados, ou definidores, como a massa e a carga elétrica. Com a partícula, podemos construir sistemas de partículas, que consistem em conjuntos de partículas com suas características definidoras.

No contexto de sistemas de partículas, ou sistemas físicos, introduz-se o conceito de interação e de movimento. Duas partículas em um mesmo sistema, no geral, interagem entre si mudando seus estados de movimento. A interação entre partículas depende dos observáveis intrínsecos às partículas do sistema, como a massa e a carga elétrica, que geram as interações gravitacional e eletromagnética, respectivamente. Estados de movimento necessitam da estrutura matemática para serem devidamente definidos, mas estamos chegando lá.

Toda característica de um sistema físico, inclusive as características intrínsecas, é um observável. O observador é um sistema físico munido de 
um aparato de medida, ou seja, uma forma de se coletar informação sobre um determinado observável do sistema físico alvo. Esta coleta de informações é denominado de medição, enquanto a informação coletada é chamada de medida. Uma medida é um elemento do conjunto de todas as medidas possíveis de serem coletadas sobre determinado observável, o espectro. Considerando-se um espectro relacionado a um observável, uma medição pode filtrar uma única medida, ou um subconjunto de medidas do espectro.

O processo de medição consiste na interação entre o observador e o sistema físico, de modo que, em via de regra, os estados de movimento de ambos podem ser alterados. Contudo, definimos uma medida clássica como aquela que perturba minimamente o estado de movimento do sistema, de modo que a interferência da medição é considerada desprezível.

\section{Sobre o mapeamento dos conceitos primitivos em estruturas matemáticas}

Para a construção de uma teoria coerente para a mecânica clássica, vamos nos utilizar da precisão matemática. Esta é a vocação primordial da física: descrever e predizer o comportamento de fenômenos naturais de forma precisa e inequívoca. Um caminho que poderíamos seguir é o de postular, através da observação e experiência, o comportamento dos conceitos primitivos da teoria. Para tanto, precisaríamos incluir outros conceitos e demonstrar seu comportamento matemático. Contudo, nosso ponto de vista será mais simples. A partir dos conceitos já estabelecidos, estabeleceremos um mapa para estruturas matemáticas já existentes. Este mapeamento tem o objetivo primeiro de estabelecer precisamente uma forma de relacionar as partículas, movimento e interações de um sistema.

O mapeamento se dá através de postulados, que relacionam conceitos físicos a objetos matemáticos. Definiremos, primeiro, o conceito de posição:

Postulado 1: A posição de uma partícula consiste em um elemento (ou ponto) do espaço euclidiano tridimensional $\mathbb{R}^{3}$. 
Neste caso, introduzimos um novo conceito, o de posição, e este conceito está vinculado a uma estrutura matemática, um espaço euclidiano. Assim, em nosso ponto de vista, o conceito de posição é um conceito derivado, necessita da teoria matemática para ser definido.

Por outro lado, definir um conceito a partir da estrutura matemática exige que a própria estrutura seja devidamente compreendida e explorada. A teoria matemática cria, neste sentido, um terreno abstrato. É neste terreno que os conceitos primitivos e derivados são posicionados e, assim, a teoria física toma vida própria.

Existem, de fato, duas estruturas matemáticas conhecidas como $\mathbb{R}^{3}$. A primeira dessas estruturas é uma variedade diferenciável; um espaço de pontos no qual é possível definir curvas suaves. Como veremos, esta propriedade será de importância fundamental para a descrição do movimento. Por outro lado, o espaço euclidiano é também um espaço vetorial. Portanto, a posição de uma partícula clássica também pode ser representada pelos tão conhecidos vetores euclidianos, com todas as suas propriedades. Trataremos dessas propriedades mais adiante.

Vamos, primeiro, tratar do espaço $\mathbb{R}^{3}$ como uma variedade. O protótipo do espaço euclidiano é a reta real $\mathbb{R}$, que consiste em uma representação do conjunto dos número reais sobre uma reta (fig 1).

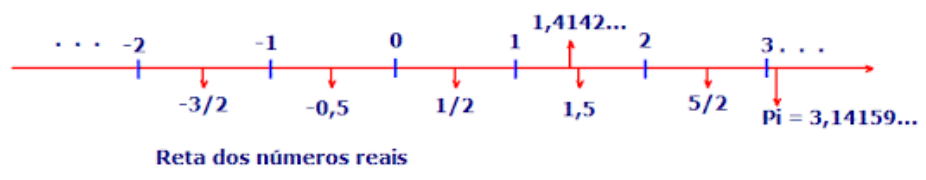

Figura 1: A reta real. De IQ-UNESP.

A reta real, por definição, é o espaço euclidiano unidimensional, ou o primeiro espaço euclidiano, denotado por $\mathbb{R}$. Como o conjunto dos números reais possui uma ordem, a reta real é um espaço ordenado e, assim, possui uma topologia natural de intervalos abertos: dois números reais $a$ e $b$, tais que $b>a$, definem intervalo aberto $(a, b) \subset \mathbb{R}$ :

$$
(a, b)=\{x \in \mathbb{R}: a<x<b\}
$$


A reta real, apesar de ilimitada, pode ser coberta por intervalos abertos, de modo que a união de abertos é um aberto, e a interseção de um número finito de abertos é também um aberto. Essas propriedades são as definidoras de um espaço topológico.

Uma segunda propriedade fundamental da reta real é a de que a ordem inerente aos números reais induz a uma forma natural de se definir a distância entre dois pontos. Esta forma é dada pela diferença absoluta, ou módulo:

$$
\forall a, b \in \mathbb{R},|a-b|= \begin{cases}a-b & a m p ; \text { se } a<b, \\ b-a & a m p ; \text { se } b<a .\end{cases}
$$

A existência desta função define o primeiro espaço euclidiano como um espaço métrico.

Existem sistemas físicos que podem ser encaixados, mesmo agora, no espaço euclidiano de uma dimensão. São aqueles vinculados a se mover sobre uma reta, como por exemplo o sistema massa-mola. Este sistema consiste em um corpo de massa $M$ preso a uma extremidade de uma mola, que por outro lado está presa a um anteparo, como no caso da fig 2.

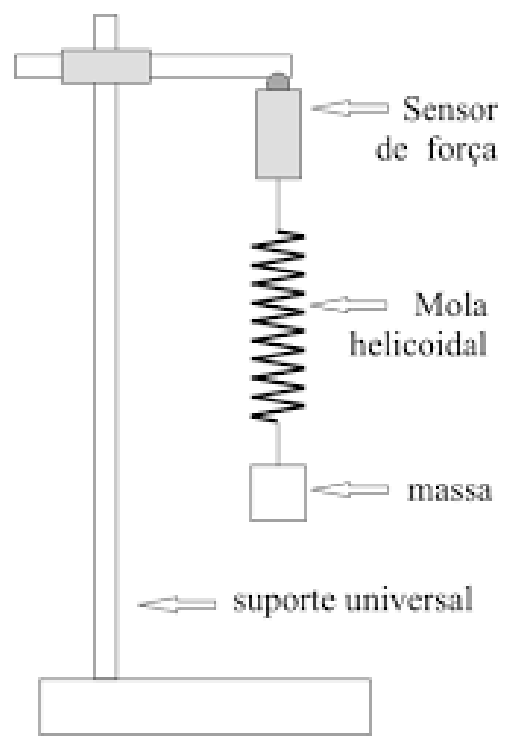

Figura 2: Sistema massa-mola. De UEL. 


\section{O produto cartesiano}

Como dissemos anteriormente, a reta real é o protótipo do espaço euclidiano. No caso de $\mathbb{R}$, este espaço é definido pelo conjunto dos números reais com a topologia da reta, ou equivalentemente, pela métrica descrita pelo módulo.

Os demais espaços euclidianos podem ser construídos a partir da reta real e de um produto cartesiano. Sejam dois pontos $a, b \in \mathbb{R}$, podemos construir uma lista ordenada $(a, b)$. Neste caso, $(a, b) \neq(b, a)$. O conjunto de todas as listas ordenadas bi-dimensionais, ou bi-ordenadas, forma um conjunto de pontos denominado espaço euclidiano bidimensional, ou simplesmente o plano cartesiano $\mathbb{R}^{2}$. Dizemos que

$$
\mathbb{R}^{2}=\mathbb{R} \otimes \mathbb{R}
$$

em que $\otimes$ denota o produto cartesiano.

Portanto, cada ponto $x \in \mathbb{R}^{2}$ é representado por uma dupla ordenada $(a, b)$, $\operatorname{com} a, b \in \mathbb{R}$. Esta relação é representada simplesmente por $x \equiv(a, b)$. Portanto, a posição de uma partícula em um sistema bi-dimensional é mapeada no plano cartesiano, em que cada ponto constitui em dois números reais ordenados. Um exemplo físico de um sistema bidimensional vem a ser o bem conhecido pêndulo simples. 


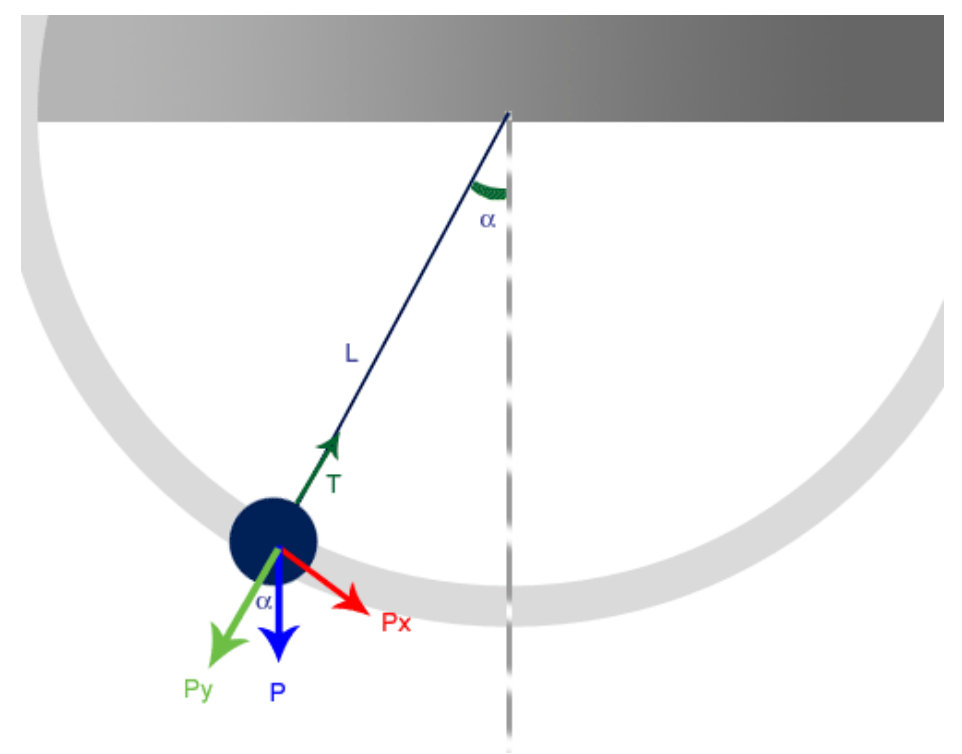

Figura 3: O pêndulo simples.

Podemos, também, definir uma forma de se medir a distância entre dois pontos de $\mathbb{R}^{2}$. Se nos reportarmos à função que faz o mesmo trabalho em $\mathbb{R}$, a função módulo, poderíamos ser tentados a utilizar a seguinte função:

$$
D(x, y) \equiv\left|x_{1}-y_{1}\right|+\left|x_{2}-y_{2}\right|,
$$

em que

$$
x, y \in \mathbb{R}^{2}: x \equiv\left(x_{1}, x_{2}\right), \quad y \equiv\left(y_{1}, y_{2}\right) .
$$

A função (??) define uma boa métrica: ela é simétrica $D(x, y)=D(y, x)$, é sempre positiva e é nula se, e somente se, $x=y$. Contudo, esta não é uma métrica aceitável fisicamente.

Para compreender por que não podemos utilizar a métrica (??) para calcular a distância entre dois pontos, devemos apelar à observação e à experiência. Na antiga Grécia, a observação da geometria plana da natureza levou ao principal resultado da geometria euclidiana: o Teorema de Pitágoras:

O Teorema de Pitágoras: Sejam $b$ e $c$ dois catetos de um 
triângulo retângulo. Neste caso, a hipotenusa $a$ é calculada por

$$
a=\sqrt{b^{2}+c^{2}}
$$

Assim como no caso unidimensional, o espaço $\mathbb{R}^{2}$ também possui uma representação gráfica, através do plano cartesiano da fig. 4.

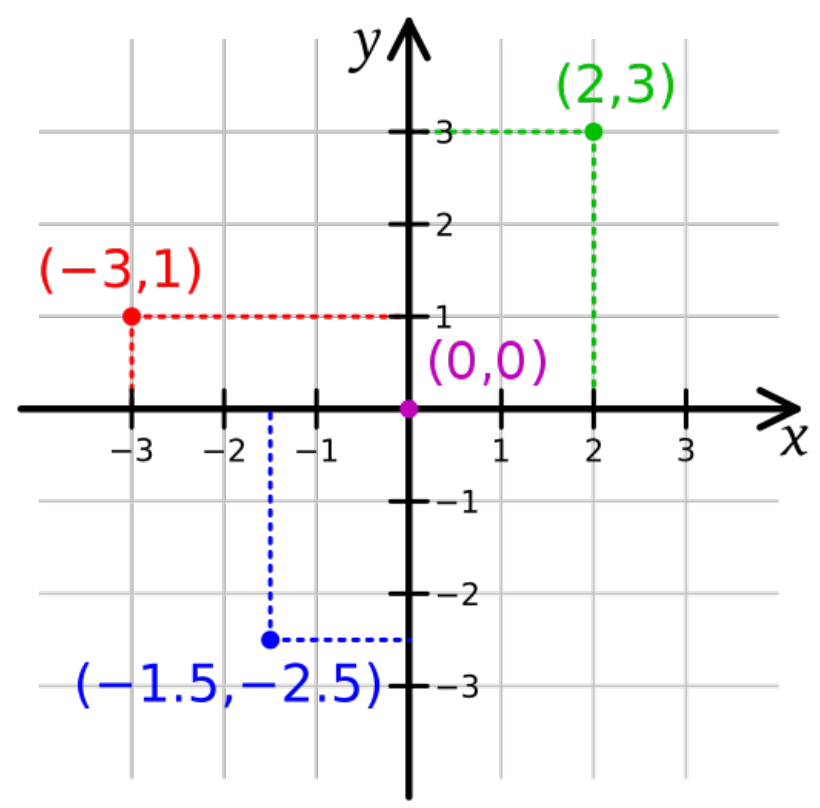

Figura 4: O plano cartesiano representado como a interseção entre duas retas reais. Neste gráfico, $x$ é a variável que representa a reta horizontal, ou coordenada abscissa. A variável y é aquela que representa a reta vertical, ou ordenada.

$D(x, y)=\sqrt{\left(y_{1}-x_{1}\right)^{2}+\left(y_{2}-x_{2}\right)^{2}}$.exemplo, se desejamos calcular a distância entre os pontos $(2,3)$ e $(-3,1)$ no plano da fig. 4 , basta desenharmos um triângulo retângulo com o cateto maior no eixo das abscissas (eixo 1 , ou eixo $x$ ), do ponto $(-3,1)$ ao ponto $(2,1)$. O cateto menor deve ser desenhado paralelo ao eixo das coordenadas (eixo 2, ou eixo y), do ponto $(2,1)$ ao ponto $(2,3)$. Neste caso, temos

$$
D=\sqrt{(2-(-3))^{2}+(3-1)^{2}}=\sqrt{29} .
$$


De forma geral, a distância euclidiana entre dois pontos $x \equiv\left(x_{1}, x_{2}\right)$ e $y \equiv\left(y_{1}, y_{2}\right)$ é calculada pela função

$$
D(x, y)=\sqrt{\left(y_{1}-x_{1}\right)^{2}+\left(y_{2}-x_{2}\right)^{2}} .
$$

\section{O espaço $\mathbb{R}^{3}$}

Com o produto cartesiano, podemos utilizar a reta real para construir espaços de dimensão maiores que 2 . O espaço que nos interessa, contudo, é o espaço euclidiano tridimensional

$$
\mathbb{R}^{3} \equiv \mathbb{R} \otimes \mathbb{R} \otimes \mathbb{R}=\mathbb{R} \otimes \mathbb{R}^{2}
$$

Os pontos do espaço $\mathbb{R}^{3}$ são representados por triplas ordenadas $(x, y, z)$ ou $\left(x_{1}, x_{2}, x_{3}\right)$, dependendo da notação utilizada sobre os eixos coordenados.

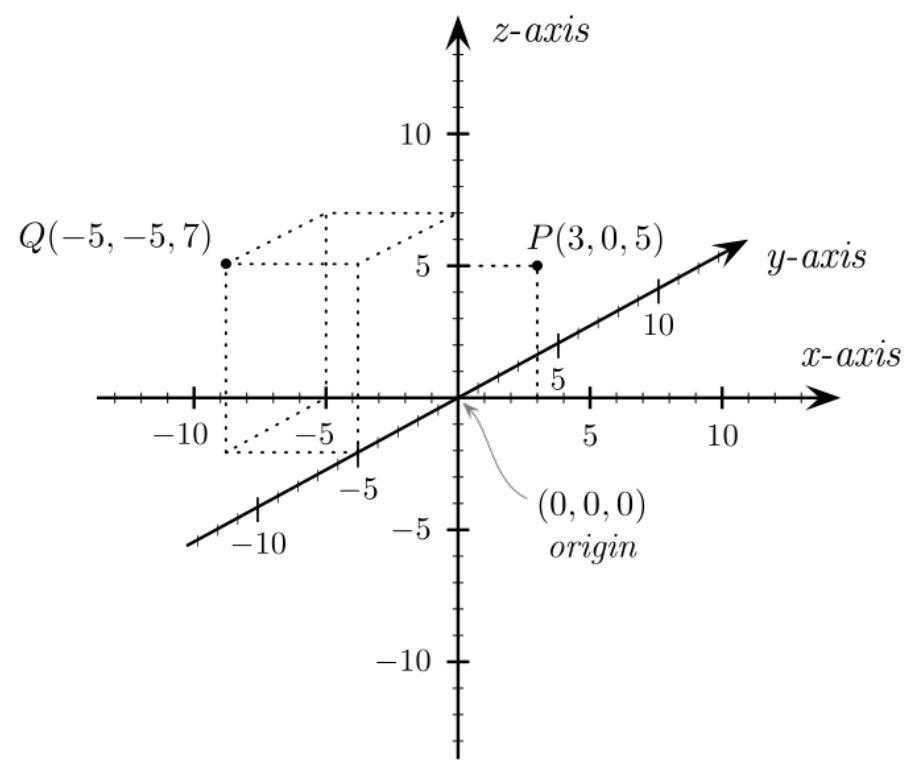

Figura 5: Representação gráfica do espaço euclidiano $\mathbb{R}^{3}$. Cada reta real é representada por um eixo coordenado, os eixos $x, y$ e $z$. Também utilizaremos as notações $\left(x_{1}, x_{2}, x_{3}\right)$ ou $\left(e_{1}, e_{2}, e_{3}\right)$ para os mesmos eixos. 


\section{A métrica euclidiana em $\mathbb{R}^{3}$}

Mais uma vez, uma métrica que possibilite calcular a distância entre dois pontos de $\mathbb{R}^{3}$ é necessária. E mais uma vez esta métrica deve ser coerente com o teorema de Pitágoras. Assim, podemos introduzir o segundo postulado:

Postulado 2: A distância entre duas partículas, cujas posições são representadas respectivamente pelos pontos $x \equiv\left(x_{1}, x_{2}, x_{3}\right)$ e $y \equiv\left(y_{1}, y_{2}, y_{3}\right)$, é dada pela hipotenusa do triângulo retângulo no qual um dos catetos é representado pela distância $\left|y_{3}-x_{3}\right|$ no eixo $e_{3}$, e o outro cateto é representado pela hipotenusa do triângulo retângulo formato pelos catetos de distância $\left|y_{1}-x_{1}\right|$, no eixo $e_{1}$, e pelo cateto de distância $\left|y_{2}-x_{2}\right|$ no eixo $e_{2}$. Neste caso,

$$
D(x, y)=\sqrt{\left(y_{1}-x_{1}\right)^{2}+\left(y_{2}-x_{2}\right)^{2}+\left(y_{3}-x_{3}\right)^{2}} .
$$

A métrica definida em (??) é um tipo de função denominado função de dois pontos. Como o espaço tem três dimensões, a métrica é uma função de seis variáveis,

$$
D(x, y)=D\left(x_{1}, x_{2}, x_{3}, y_{1}, y_{2}, y_{3}\right)
$$

Esta função obedece às propriedades de uma boa métrica:

1. Simetria: $D(x, y)=D(y, x)$;

2. Positividade: $D(x, y) \geq 0$;

3. Não degenerescência: $D(x, y)=0$ se, e somente se, $x=y$. 